\title{
The clinically validated viral superinfection therapy (SIT) platform technology could cure early cases of COVID-19 disease
}

Imre Kovesdi, $\mathrm{PhD}^{1,8}$, Volker Sandig, $\mathrm{PhD}^{2}$, Shimon Slavin, $\mathrm{MD}^{3}$, Wolfgang Renz, $\mathrm{MD}^{4}$, Marc Van Ranst, $\mathrm{PhD}^{5}$, Peter M. Chumakov, MD, PhD, $\mathrm{DSc}^{6,7}$, and Tibor Bakacs, MD, PhD, $\mathrm{DSc}^{8^{*}}$

${ }^{1}$ DNAtrix Inc, 2450 Holcombe Blvd. Suite X+ 200, Houston, TX 77021, USA; Tel: +1-301963-1641, Email: imkovesdi@gmail.com;

${ }^{2}$ ProBioGen AG, Standort HBS Herbert-Bayer-Straße 8, 13086 Berlin, Germany; Tel: +49 30 322935 - 300; e-mail: Volker.Sandig@ probiogen.de;

${ }^{3}$ Scientific \& Medical Director, Biotherapy International, Tel Aviv, Israel and Medical Director, HepC Therapeutics, Budapest, Hungary; Tel.: +972 54942 5456; Email: slavinmd@gmail.com;

${ }^{4}$ Adj. Professor of General Surgery McGill University, Montreal, Canada; Email: wolfgang.renz@mcgill.ca;

${ }^{5}$ Laboratory of Clinical Virology, Rega Institute, Herestraat 49 box 1040, 3000 Leuven, Belgium; Tel: +32-16-347 908, Email: vanranstmarc@gmail.com;

${ }^{6}$ Engelhardt Institute of Molecular Biology, Russian Academy of Sciences; 32, Vaviolva str., Moscow, 119991, Russia; Tel: +7-495-1352311; e-mail: chumakovpm@yahoo.com; peterchumakov@gmail.com;

${ }^{7}$ Chumakov Federal Scientific Center for Research and Development of Immune and Biological Products, Russian Academy of Sciences, Moscow, Russia; 108819;

${ }^{8}$ HepC, Inc, 1012 Budapest, Miko str. 3. II. fl. 11., Hungary; Tel: +36-30-726-5122, Email: tiborbakacs@gmail.com; http://www.superinfectiontherapy.com/;

* Corresponding author: Tibor Bakacs, MD, PhD, DSc

\section{ABSTRACT}

Currently, SARS-CoV-2 infection which is the causative agent for COVID-19 disease is a worldwide pandemic with more than 100 million global cases and more than 2.0 million deaths (as of January, 2021). While several vaccines for prevention of COVID-19 have already been registered by the regulatory authorities, the problem is that the substitution rate of this virus is estimated to be one change per 2 weeks, thus mutations could arise that threaten the efficacy of 
vaccines. Unfortunately, there is no current evidence from random clinical trials to recommend any specific post-exposure treatment for patients with suspected or confirmed COVID-19 disease. Here we propose an innovative superinfection therapeutic (SIT) strategy, which could complement the development of prophylactic vaccines. SIT is based on clinical observations that unrelated harmless viruses might interact in patients infected with pathogenic virus. During SIT, the patient benefits from superinfection with an apathogenic double-stranded RNA (dsRNA) virus such as the infectious bursal disease virus (IBDV), which is a powerful activator of the interferon-dependent antiviral gene program. An attenuated vaccine strain of IBDV was already successfully administered to resolve acute and persistent infections induced by two completely different viruses, the hepatitis B (DNA) and C (RNA) viruses (HBV/HCV). The safety of orally administered acid-resistant IBDV strain R903/78 reverse engineered viral drug candidate was demonstrated in 10 stage IV cancer patients who exhausted all conventional therapy. Following repeated oral administration of the virus up to $10^{9}$ infectious units (IU)/ dose, only mild flu-like side effects were reported in some patients. Proof-of-principle efficacy was demonstrated in an early COVID-19 patient who was successfully treated with $3 \times 10^{6}$ IU of an attenuated IBDV vaccine. A small scale dose-finding Phase I safety study is proposed.

Keywords: Coronavirus, SARS-CoV-2, COVID-19, interferon, IFN, superinfection therapy, SIT, infectious bursal disease virus, IBDV, R903/78, safety, treatment.

\section{Introduction}

2020 has witnessed the development of close to 60 vaccine candidates against SARS-CoV-2, some having more than 90\% efficacy to prevent COVID-19 disease in clinical trials (Knoll and Wonodi, 2020). The problem, however, is that the substitution rate of SARS-CoV-2 is estimated to be $1.13 \times 10^{-3}$ substitutions per site per year in the human population, which is around one change per 2 weeks (Candido et al., 2020). Therefore, vaccines might not be sufficient alone to solve the current and future pandemics. A good example is the explosive spread of a dangerous strain of SARS-CoV-2 virus in minks and several documented cases of mink-to-human transmission (Oude Munnink et al., 2021). The new strain forced the culling of 17 million minks in Denmark, the world's largest mink producer in order to prevent farms become a permanent virus reservoir that might jeopardize vaccines. Even more alarming is another new variant (B.1.1.7) that has been found in England, which caused cases to soar. This variant (with many 
mutations, including eight in the crucial spike protein) has quickly replaced other variants, its frequency rose from $0.2 \%$ to $2.3 \%$ in 3 weeks as a result of exponential growth (Kupferschmidt, 2021). The B.1.1.7 variant may be the harbinger of a new, and unpredictable pandemic phase as a result of viral evolution. Already other worrying variants have emerged (e.g. 501Y.V2 in South Africa). A receptor-binding-domain mutation, called E484K and several other mutations can, to varying degrees, escape recognition by antibodies in peoples' convalescent sera (Callaway, 2021). Eventually, mutations could arise that threaten the efficacy of vaccines. Since there is no current evidence from random clinical trials to recommend any specific treatment for patients with suspected or confirmed COVID-19 disease, and considering the fact that even properly vaccinated individuals and patients recovering from COVID-19 may still be infected with SARSCoV-2, a broad spectrum antiviral therapy is urgently needed.

Here we propose an innovative safe therapeutic strategy, which could complement the development of prophylactic vaccines (Bakacs et al., 2018; Kovesdi and Bakacs, 2019). The clinically validated viral superinfection therapy (SIT) platform technology may contribute to our current and future pandemic preparedness. SIT is an entirely different modality from the antiviral drugs currently in pipelines as it exploits an apathogenic dsRNA viral vector (infectious bursal diseases virus; IBDV strain R903/78) that activates the interferon-dependent antiviral gene program of the human body. IFNs are the most potent innate protection for immunologically naïve people but this response is declining rapidly with age (Agrawal, 2013).

SIT is a therapeutic approach where a non-pathogenic virus is used to infect patients harboring a pathogenic virus and so reduce or eliminate the disease manifestations of pathogenic virus. An attenuated vaccine strain of IBDV was already successfully administered to resolve infections induced by two completely different viruses, the hepatitis B (DNA) and C (RNA) viruses (HBV/HCV) in acute and decompensated chronic hepatitis patients (Csatary et al., 1998) (Csatary et al., 1999). Importantly, this virus is also a potential vaccine vector drug candidate, since a recombinant IBDV was previously generated that displays exogenous viral peptides from a replication competent IBDV (Upadhyay et al., 2011). The epidemiological efficacy of a similar strategy to SIT using attenuated vaccine viruses that stimulate the production of endogenous interferon (IFN) of the host had already been tested in large scale clinical trials (Chumakov et al., 1992) as described below. 
In the current work, we propose a mechanism of action for SIT (Figure 1), we characterize the in vitro interferon response generated by IBDV. We also present safety results obtained in advanced cancer patients with high dose of IBDV and a case study of an early COVID-19 patient who was successfully treated with low dose of IBDV.

Historical background: oral administration of live attenuated vaccines reduced disease incidence of acute respiratory diseases

More than 110 types of viruses are now being associated with acute respiratory infections (ARI). A completely new mode of prevention was proposed targeting all these viral infections during influenza outbreaks and other associated acute respiratory infections. The principle was based on the use of standard live enterovirus vaccines that stimulate the production of endogenous interferon of the host. To this end, controlled trials of epidemiological efficacy were carried out during three seasonal outbreaks of influenza and other associated acute respiratory infections (between 1968-1971) in 16 regions of three republics in the former Soviet Union (Chumakov et al., 1992). The surveillance covered about 320,000 people, $2 / 3$ of whom have orally received live enteroviral interferon-inducing vaccine strains (LEV) 2-3 times. No adverse reactions were observed following the administration of LEV. The enterovirus vaccines provided protection from symptoms for at least 22,700 people from influenza and ARIs out of 152,042 individuals by reducing the incidence on average by 3.2 fold compared to controls who did not receive LEV. Administration of enterovirus vaccines 2-3 times at intervals of 10-14 days provided 3-5 weeks protection against influenza and acute respiratory infections. Oral administration (in liquid form, or as jelly beans) of the interferon-inducing enterovirus vaccines was the most convenient, but intranasal delivery was also effective. Typically, a dose of vaccine contained 1 million culture infectious units of the virus. The post-infection administration of standard LEV at the beginning of outbreaks of influenza and ARI had a therapeutic effect ameliorating the disease. Importantly, in 12 patients the symptoms of the disease were abolished within 10-12 hours after taking the vaccine. In 6 individuals a significant improvement was observed within 10-12 hours, while the symptoms completely disappeared within 24 hours. Recently, Chumakov et al proposed that live attenuated vaccines in general, and oral poliovirus vaccine (OPV) in particular, could provide temporary protection against COVID-19 by the interferon-mediated stimulation of innate immunity (Chumakov et al., 2020). The authors stated that this strategy may even have an 
advantage over specific vaccines if SARS-CoV-2 undergoes mutations that could lead to loss of vaccine efficacy.

\section{A testable hypothesis}

Corticosteroids and antibodies are treatment options for critically ill patients with COVID-19 disease requiring hospitalization. However, interventions that can be administered early to prevent disease progression are urgently needed. Such treatment should be dosed without requiring intravenous infusions and should be available widely at low cost (Kim et al., 2020). Therefore, we propose the use of the clinically tested innovative post-exposure SIT in cases of early COVID-19 disease. We expect that by preventing disease progression, hospitalization and longer-term complications could be substantially reduced. Since interferon is active against most vertebrate-infecting viruses, SIT could be developed into the first biologic drug candidate, for a new broad-spectrum antiviral "one drug, multiple bugs" treatment approach of viral diseases the Science called for (Bekerman and Einav, 2015).

The SIT technological platform is clearly different from existing approaches that could be scaled up rapidly at low-cost with a strong likelihood of achieving a substantial impact in the near term. Therefore, SIT could be quickly developed into an easy-to-produce and nasally or orally administered therapeutic vaccine to alleviate the deadly lag phase of surge capacity in vaccine production in a pandemic. It is simple to administer and feasible even in resource limited settings.

Because of its major economic importance to the world's poultry industries, attenuated IBDV strains are used as commercial vaccines for treatment in chicken farms. These vaccines have an excellent safety record. Despite its worldwide distribution, IBDV is not known to be a hazard in transmitting to any other species than domestic fowl. Furthermore, the vaccine strain of our IBDV drug candidate (R903/78) is harmless in chickens as well.

Importantly, IBDV is one of the most stable viruses known that can survive significant temperature variations, which allows for transportation and storage without dry ice. It can endure extreme acidic environments permitting oral delivery. These characteristics would alleviate undeniable logistical challenges of therapeutic vaccine production, transport and cost. 
Our primary goal is to leverage our clinically tested IBDV-SIT technology platform for the safe, effective, widely affordable post-infection treatment of early COVID-19 patients, even in resource limited settings. Our major goal is, therefore, to provide the R903/78 drug substance for use in early COVID-19 patients for evaluation. We believe that the R903/78 viral drug candidate could be produced for use in early COVID-19 patients within few months for evaluation.

Easy reproducible production of a reverse engineered virus: $R 903 / 78$ drug candidate

To address safety concerns a tissue culture adapted IBDV vaccine strain V903/78 was cloned into cDNA plasmids ensuring easy reproducible production of a reverse engineered virus R903/78. The therapeutic drug candidate was extensively characterized by immunocytochemistry assay (ICC), virus particle determination, and immunoblot analysis. The biodistribution and immunogenicity of the IBDV agent was determined in mice, which is not a natural host of this virus, by quantitative detection of IBDV RNA with qRT-PCR and virus neutralization test (VNT), respectively. The virus was stable at a wide $\mathrm{pH}$ range, demonstrating significant resistance to low $\mathrm{pH}$ and also proved to be highly resistant to high temperatures. No pathological effects were observed in mice and rats, respectively. Single and multiple oral administration of IBDV elicited a neutralizing antibody response that peaked around 21 days post-infection. Repeat oral administration of R903/78 was successful despite the presence of neutralizing antibodies. Viral replication could not be demonstrated in mice and rats, but the powerful induction of IFN related genes was observed. Several human cell lines supported IBDV propagation in the absence of visible cytopathic effect (CPE) and was secreted into the medium. This resulted in the development of a simple downstream manufacturing process. Several lots of agent were manufactured under research and GLP conditions. Also, different formulations were tested for stability and a formulation that was stable at $5^{\circ} \mathrm{C}$ for 6 months was developed. These preclinical data support the development of an orally or nasally delivered anti-viral agent for human use (Hornyak et al., 2015).

\section{Simplifying the manufacturing process to achieve higher yields at lower costs}

Simplification of the manufacturing processes and the achievement of higher yields could rapidly increase production to provide drug substance in larger quantities. To this end, previous productions in iCELLis bioreactor using Vero cells will be changed to production in serum free and chemically defined media using the Wave bioreactor and ProBioGen's AGE1.CR cell line 
family. AGE1.CR and AGE1.CR.PIX cells proliferate in suspension in commercially available, chemically defined medium free of animal-derived components and allows robust and fully scalable proliferation in different bioreactor systems. AGE1.CR cell line family is designed to meet health regulatory guidelines and the 'defined risk' concept issued by the FDA for generation of new cell substrates for application in the production of live vaccines because the immortalizing genes are known and traceable (Jordan et al., 2009). It was demonstrated that both cell lines AGE1.CR and AGE1.CR.PIX are fully permissive for IBDV strain R903/78. Titers up to $10^{10} \mathrm{TCID}_{50} / \mathrm{ml}$ can be achieved in a 4-day process, more than 1000 times higher than in VERO cells, which secures a good yield for a commercially viable virus production.

IBDV strongly induces IFN-beta in vitro

The human lung epithelial cells (A549) are fully susceptible to infection with IBDV and a primary cell line to study IFN responses. To study whether type I (IFN-alpha, IFN-beta), type II (IFN-gamma) and type III (IFN-lambda) are induced by IBDV strain R903/78, A549 cells were infected at different multiplicity of infections (MOI) $(0.01,0.1$ and 1$)$ for $32 \mathrm{~h}$. The expression levels of the different interferons (IFN-beta, IFN-gamma, IFN-lambda) and interferon-regulated genes (human myxovirus resistance protein-A $[\mathrm{MxA}]$ and the human interferon-stimulated gene 56 [ISG56]) were analyzed by a quantitative one-step RT-PCR. The relative expression levels were normalized against non-infected cells. Glyceraldehyde-3-phosphate dehydrogenase (GAPDH) was used as an internal standard. As shown in Figure 2, IBDV strongly induces IFNbeta in cells infected for $32 \mathrm{~h}$ with IBDV at MOI=1 (about 30x) and IFN-lambda (5-10x). Surprisingly, in contrast to other viruses (Adenovirus, Encephalomyocarditis virus) IFN-gamma is not induced in A549 cells. Genes downstream of IFN were not yet strongly induced, likely due to an early harvest time. Furthermore, IBDV does not lyse the A549 cells. In contrast to IBDV, Encephalomyocarditis virus (EMCV) used as a control induces IFN-gamma dominantly. Values are higher for EMCV if calculated relative to GAPDH, because EMCV lyse cells efficiently and there are almost no live cells remaining that could provide GAPDH mRNA (data not shown).

IFNs are the most potent innate protection when we are immunologically naïve and this response is declining rapidly in aged people. SARS-CoV-2 is extremely sensitive to type I IFNs. For instance, in Vero cells, IFN alpha treatment reduces SARS-CoV-2 titers by 3-4 orders of magnitude (Lokugamage et al., 2020) which would indicate that the interferon activation 
properties of R903/78 are promising to counter SARS-CoV-2 virus infections. Systemic interferon treatment is associated with severe side effects. Higher toxicity of IFN-gamma compared to IFN-alpha and -beta limits its clinical use. IFN-lambda is a potent inducer of innate response against viruses and is associated with less side effects. Therefore, the natural specific induction profile of IBDV maximizes antiviral action with minimal side effects, thus opening the therapeutic window.

Preliminary safety study of R903/78 viral drug candidate in stage IV cancer patients resistant to all conventional therapies

The safety of the R903/78 virus was first tested in 10 stage IV cancer patients with different type of cancers ( 2 cases of recurrent glioblastoma multiforme, cholangiocarcinoma, breast cancer, rectal cancer, primary tongue carcinoma, oropharynx cancer, malignant melanoma, prostate cancer and multiple myeloma). These patients were resistant to all conventional therapy. The rationale for developing our R903/78 viral drug candidate also as an anticancer modality is twofold. First, oncolytic viruses that are inducing IFNs are currently undergoing clinical trials against melanoma, glioma, pancreatic, and breast and other cancers. Oncolytic viruses with potential therapeutics for cancer patients are based on adenoviruses, Herpes Simplex Viruses, Vaccinia viruses, Reoviruses, viruses of the Paramyxoviridae family, Picornaviruses, and retroviral replicating vectors. Second, some cancer cells have lower capacity to activate interferon secretion, rendering them higher susceptibility to viral infection, thus with potentially preferred targeting into malignant cells. However, there is still an unmet need for effective and inexpensive, easy to administer, transport and storage stable and safe cancer therapeutics. Viruses of the Birnaviridae family promote immunogenic cell death and immunomodulation (Qin and Zheng, 2017) and, thus, may allow an efficient treatment of a broad range of different cancer types. The well-known strong type I IFN response induced by IBDV triggers both innate and adaptive immune responses that may result in a proinflammatory tumor microenvironment which contributes to an antitumor response.

After signing an informed consent oral IBDV R903/78 viral treatment of cancer patients started with a dose of $10^{6}$ infectious units/ dose. Then, increments from $10^{6}$ to $10^{7}$ to $10^{8}$ and to $10^{9} \mathrm{IU} /$ dose were orally administered. Each dose was given a week apart in order to monitor patients for potential side effects. It was reassuring that even very high doses of R903/78 virus ( $\left.10^{9} \mathrm{IU} / \mathrm{dose}\right)$ 
were associated with only mild flu-like side effects, which occurred usually 2-3 days after treatment. While not all patients reported side effects, some complained about fatigue, sore thigh, legs, bones and muscles, and sweating. Body temperature increase occurred on day 2 (never exceeded $38.5^{\circ} \mathrm{C}$ ) and then decreased by 0.5 degrees per day to normal levels. The peak of the symptoms was at day 3 and they completely disappeared by day 5 .

In conclusion, with only mild side effects at the highest $10^{9} \mathrm{IU} /$ dose, we expect no safety problems for the COVID-19 clinical trials. The Phase I trial is proposed to start at $10^{6} \mathrm{IU} / \mathrm{dose}$ and the Phase II trial expected to be done at $10^{7} \mathrm{IU} / \mathrm{dose}$ (see discussions below).

Proof-of-principle of the R903/78 viral superinfection treatment of an early COVID-19 patient with mild symptoms

A 43 year old male patient ${ }^{1}$ reported extreme muscle pains, shivering, tiredness, and headaches. While the pains almost disappeared by the 3rd day, constant headache and dizziness, and a very low energy to move around remained. When the patient would stand up felt very dizzy, as almost ready to lose his balance. By the end of the first week of symptoms, the patient complained about a decrease in his ability to smell odors in and around the house. Six days after the first symptoms a government sponsored COVID-19 PCR test proved to be positive. Then oral IBDV treatment was started with $10^{6} \mathrm{IU}$ of an attenuated IBDV vaccine virus in the evening, after dinner. This is the lowest dose proposed for the Phase I clinical trial. By the next day morning the patient reported that his headaches have lessened, and the dizziness and unsteadiness when standing up has also been less prominent. Overall, there was a higher level of motivation to do physical activity, like walking in the house and moving around. The second oral IBDV with $10^{6}$ IU of virus was again administered next day in the evening, after dinner. The patient reported that after the second vial treatment, he had a very good night of sleep, for the first time without any symptoms, or pains, with no headaches and or muscle aches. By morning he woke up with more energy without dizzy feelings or headaches. He was able sit in front of the computer and read mails, articles, think and write without any problems. Before the viral treatment all these activities were difficult, as he had felt a loss of short term memory, and a really difficult time to concentrate on any intellectual tasks, as simple as just paying an invoice online. Also, after the second treatment dose he felt motivated to do some minor physical exercise, like slow and easy

\footnotetext{
${ }^{1}$ The treatment was conducted by the senior author (Tibor Bakacs, M.D.).
} 
stationary cycling and also light gymnastics exercising. The third oral IBDV dose with $10^{6}$ IU of virus was administered next day also in the evening, after dinner. After the 3rd viral dose, he felt more motivated physically, even able to do a more intensive home based exercise for the first time since he started with the COVID-19 symptoms. This would mean a more moderate paced stationary cycling and light weight lifting exercises at home. One other thing to note was that his sense of smell, which deteriorated in the first few days after the first COVID-19 symptoms appeared, came back. He could smell the candles in the house, already from a distance, which before he was unable to do. This is reassuring since half to three-quarter of people with COVID19 disease who had olfactory dysfunction reported that they recovered their sense of smell after a month, while many others reported only an improvement. However, some people improve slowly over a long period (Marshall, 2021). The only side effect the patient reported was a mild heartburn in his throat and in the food pipe, which lasted for three days. Three weeks after the oral IBDV superinfection therapy the patient's serum was tested for the presence of IBDV neutralizing antibodies by virus neutralization as described earlier (Hornyak et al., 2015). The highest serum dilution that inhibited plaque formation was found to be $1 / 32$ demonstrating the presence of anti-IBDV antibodies. This confirmed the results obtained in the animal model that following oral administration of IBDV neutralizing antibodies were induced. Considering that humans are not a natural host of IBDV, appearance of neutralizing antibodies after oral administration of the virus is not trivial. To our best knowledge, this is the first early COVID-19 patient with mild symptoms who was successfully treated by IBDV superinfection therapy. This single case should however be confirmed in a small scale dose-finding Phase I safety study as described below.

In conclusion, after about a week of the appearance of first symptoms, three low dose IBDV treatments alleviated most of the COVID-19 symptoms of this patent. Please note that the dose and efficacy of IBDV drug candidate in this COVID-19 patient was very similar to that of the post-infection administration of the live enteroviral interferon-inducing vaccine strains (LEV) (Chumakov et al., 1992).

\section{Planned Phase I safety study}

The Phase I safety clinical trial of the drug candidate will be conducted in 21 early COVID-19 patients with mild to moderate symptoms. The primary objective is to confirm the safety profile 
of the R903/78 product in patients with early COVID-19 disease. The safety of R903/78 will be determined by assessing adverse events and safety laboratory factors. The secondary objectives which will require higher number of patients exposed to SARS-CoV-2 are to determine the efficacy of the R903/78 product in eliminating SARS-CoV-2 virus infection and COVID-19 symptoms in patients with early COVID-19 disease. The effect of R903/78 will be determined on each of the following factors: immune assays including IFN panel, cytokine panel, T cell, NK cell and macrophage assays; anti-SARS-CoV-2 and anti-IBDV antibody tests; PCR for SARSCoV-2 and IBDV, respectively. R903/78 drug candidate containing three different doses, $1.0 \mathrm{x}$ $10^{6} \mathrm{IU}$ of IBDV (low dose group), $1.0 \times 10^{7} \mathrm{IU}$ (intermediate dose group) and $1.0 \times 10^{8} \mathrm{IU}$ (high dose group) will be administered orally, daily for 1 week.

We are developing an orally or nasally administered attenuated IBDV drug candidate (R903/78) for the treatment of early COVID-19 patients. We propose therefore an accelerated program to manufacture sufficient experimental R903/78 drug substance to treat approximately 21 patients with early disease in a Phase I clinical trial for 1 week with 1 vial/day/1 $110^{6} \mathrm{IU}$ to $1 \times 10^{8} \mathrm{IU}$ infectious units of virus and a Phase II clinical trial with approximately 100 patients at risk with a dose determined in the Phase I trial. This effort would take only a few months depending on the release testing required and allowed by EMA and/or FDA. The vials would be shipped to early COVID-19 patients to evaluate the percent of patient recovery from COVID-19. Following the successful Phase I and Phase II clinical studies, we will manufacture our drug candidate in sufficiently large scale to provide therapy for one million infected patients at risk.

Conflict of Interest: I.K., S.S., and T.B. are shareholders of HepC, Inc., V.S. is listed as an inventor on patents that protects the AGE1.CR cell line family (WO 2005/042728 and WO2007/054516)

\section{References}

Agrawal, A. (2013). Mechanisms and implications of age-associated impaired innate interferon secretion by dendritic cells: a mini-review. Gerontology 59, 421-426.

Bakacs, T., Safadi, R., and Kovesdi, I. (2018). Post-infection viral superinfection technology could treat HBV and HCV patients with unmet needs. Hepatol Med Policy 3, 2. 
Bekerman, E., and Einav, S. (2015). Infectious disease. Combating emerging viral threats. Science $348,282-283$.

Callaway, E. (2021). Could new COVID variants undermine vaccines? Labs scramble to find out. Nature 589, 177-178.

Candido, D.S., Claro, I.M., de Jesus, J.G., Souza, W.M., Moreira, F.R.R., Dellicour, S., Mellan, T.A., du Plessis, L., Pereira, R.H.M., Sales, F.C.S., et al. (2020). Evolution and epidemic spread of SARS-CoV-2 in Brazil. Science 369, 1255-1260.

Chumakov, K., Benn, C.S., Aaby, P., Kottilil, S., and Gallo, R. (2020). Can existing live vaccines prevent COVID-19? Science $368,1187-1188$.

Chumakov, M.P., Voroshilova, M.K., Antsupova, A.S., Boiko, V.M., Blinova, M.I., Priimiagi, L.S., Rodin, V.I., Seibil, V.B., Siniak, K.M., Smorodintsev, A.A., et al. (1992). [Live enteroviral vaccines for the emergency nonspecific prevention of mass respiratory diseases during fallwinter epidemics of influenza and acute respiratory diseases]. Zh Mikrobiol Epidemiol Immunobiol, 37-40.

Csatary, L.K., Schnabel, R., and Bakacs, T. (1999). Successful treatment of decompensated chronic viral hepatitis by bursal disease virus vaccine. Anticancer Res 19, 629-633.

Csatary, L.K., Telegdy, L., Gergely, P., Bodey, B., and Bakacs, T. (1998). Preliminary report of a controlled trial of MTH-68/B virus vaccine treatment in acute B and C hepatitis: a phase II study. Anticancer Res 18, 1279-1282.

Hornyak, A., Lipinski, K.S., Bakonyi, T., Forgach, P., Horvath, E., Farsang, A., Hedley, S.J., Palya, V., Bakacs, T., and Kovesdi, I. (2015). Effective multiple oral administration of reverse genetics engineered infectious bursal disease virus in mice in the presence of neutralizing antibodies. J Gene Med 17, 116-131.

Jordan, I., Vos, A., Beilfuss, S., Neubert, A., Breul, S., and Sandig, V. (2009). An avian cell line designed for production of highly attenuated viruses. Vaccine 27, 748-756.

Kim, P.S., Read, S.W., and Fauci, A.S. (2020). Therapy for Early COVID-19: A Critical Need. JAMA 324, 2149-2150. 
Knoll, M.D., and Wonodi, C. (2020). Oxford-AstraZeneca COVID-19 vaccine efficacy. The Lancet.

Kovesdi, I., and Bakacs, T. (2019). Therapeutic exploitation of viral interference. Infectious Disorders - Drug Targets 19, 1-1.

Kupferschmidt, K. (2021). Viral evolution may herald new pandemic phase. Science 371, 108109.

Lokugamage, K.G., Schindewolf, C., and Menachery, V.D. (2020). SARS-CoV-2 sensitive to type I interferon pretreatment. bioRxiv, 2020.2003.2007.982264.

Marshall, M. (2021). COVID's toll on smell and taste: what scientists do and don't know. Nature.

Oude Munnink, B.B., Sikkema, R.S., Nieuwenhuijse, D.F., Molenaar, R.J., Munger, E., Molenkamp, R., van der Spek, A., Tolsma, P., Rietveld, A., Brouwer, M., et al. (2021).

Transmission of SARS-CoV-2 on mink farms between humans and mink and back to humans. Science $371,172-177$.

Qin, Y., and Zheng, S.J. (2017). Infectious Bursal Disease Virus-Host Interactions: Multifunctional Viral Proteins that Perform Multiple and Differing Jobs. Int J Mol Sci 18.

Upadhyay, C., Ammayappan, A., Patel, D., Kovesdi, I., and Vakharia, V.N. (2011).

Recombinant infectious bursal disease virus carrying hepatitis C virus epitopes. J Virol 85, 14081414. 


\section{Figures}

Figure 1. Rationale: Superinfection with a harmless virus to induce innate antiviral host defense. In a naïve population innate immunity represents the only protection against a virus. The Avibirnavirus IBDV (Gumboro) is a common poultry pathogen. It infects B cell precursors in young chickens. Vaccine strains are safely used in mass vaccinations for $50+$ years.
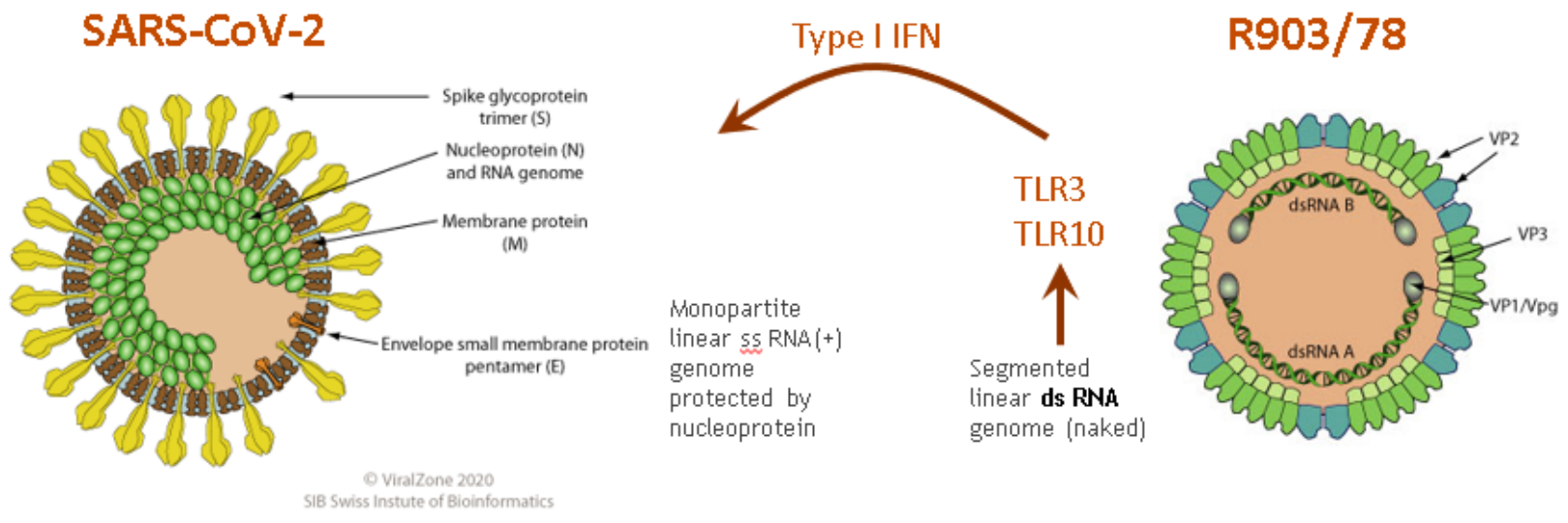
Figure 2. In vitro efficacy study of R903/78. IBDV and EMCV induce expression of interferons differently. R903/78 (IBDV) specifically induces IFN-beta and -lambda mRNA in the human lung adenocarcinoma cell line A549 (typically used for IFN induction studies). During this infection process, the A549 monolayer stays intact, GAPDH expression remains unchanged. IBDV is not lytic, in most cells virus particles are budding from the cell surface.

Encephalomyocarditis virus (EMCV) used as a control induces IFN-gamma dominantly. (Values are higher for EMCV if calculated relative to GAPDH. However, EMCV kills efficiently and therefore hardly any live cells remain that could provide GAPDH mRNA; data not shown).

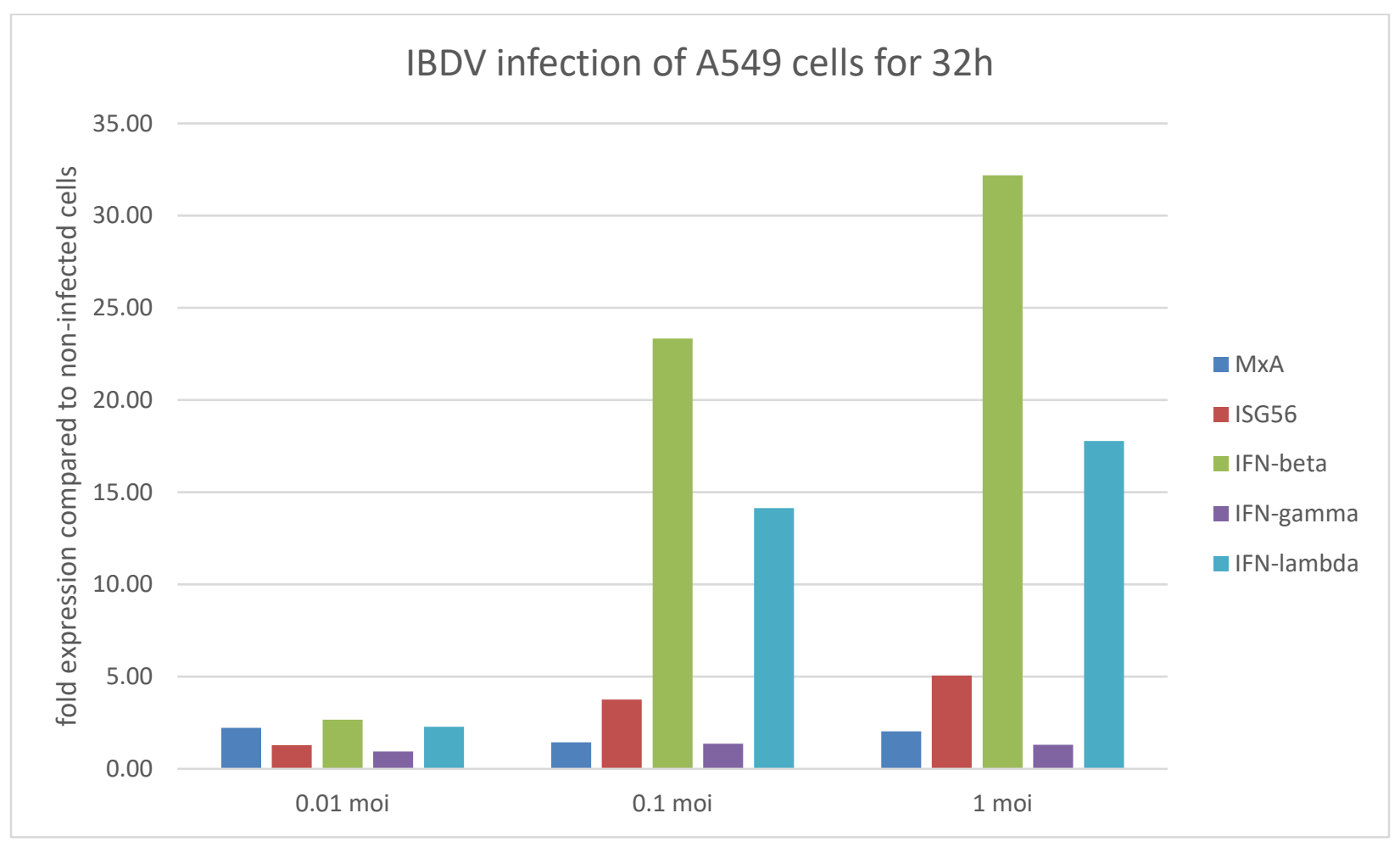

